\title{
HABILIDADES AUDITIVAS POR MEIO DA AVALIAÇÃO SIMPLIFICADA DO PROCESSAMENTO AUDITIVO EM ESCOLARES
}

\section{HEARING ABILITIES BY SIMPLIFIED AUDITORY PROCESSING TEST IN SCHOOLCHILDREN}

\author{
Gabrielle Fernand Garrido Camargo ${ }^{1}$, Jenane Topanotti ${ }^{2}$, Karlla Cassol ${ }^{2}$, Giovana \\ Romero ${ }^{2}$, Vandriéle Herber ${ }^{2}$, Aline Aparecida Tomiasi ${ }^{2}$ \\ ${ }^{1}$ Acadêmica do Curso de Fonoaudiologia, Centro Universitário Fundação Assis Gurgacz (FAG). \\ ${ }^{2}$ Docente do Curso de Fonoaudiologia, Centro Universitário Fundação Assis Gurgacz (FAG). \\ * Autor correspondente: fonojenane@gmail.com, https://orcid.org/0000-0002-2799-4135
}

\begin{abstract}
RESUMO
Introdução: Os primeiros anos da vida de uma criança são críticos para o desenvolvimento da fala e da linguagem, sendo fundamentais para a sociabilidade do ser humano. Neste período desenvolvem-se as habilidades auditivas devido à grande maturação e a plasticidade auditiva do sistema nervoso central. Desta forma, um bom desenvolvimento das habilidades auditivas será favorável no processo de aprendizagem em pré-escolares e escolares. Objetivo: Investigar as habilidades auditivas dos escolares do primeiro e segundo ano do Ensino Fundamental de nove anos por meio do desempenho nos testes da ASPA. Metodologia: A casuística foi composta por 67 escolares matriculados regularmente nos anos iniciais na Escola Municipal Hermes Vezzaro no Município de Cascavel-PR, divididos em dois grupos. O grupo 1 (G1), formado por 51 escolares do primeiro ano, sendo 30 do gênero feminino e 21 do gênero masculino, com 6 anos de idade. O grupo 2 (G2), composto por 16 escolares do segundo ano, sendo 10 do gênero feminino e 6 do gênero masculino, com 7 anos de idade. Todos foram submetidos aos seguintes procedimentos: anamnese, inspeção visual do Meato Acústico Externo, triagem audiométrica e os testes que compõem a Avaliação Simplificada do Processamento Auditivo (ASPA). Resultado: Os escolares de ambos os grupos apresentaram desempenho satisfatório em todos os testes mostrando, em sua maioria, habilidades de atenção, localização sonora e memória sequencial. No entanto, o G1 ofereceu respostas inferiores quando comparados ao G2. As crianças mais jovens tiveram maior ocorrência de alterações no teste de Memória Sequencial para Sons Não Verbais. Conclusão: A maioria dos escolares estudados apresentou as habilidades de atenção, localização sonora e memória sequencial adequadas para a faixa etária. A ASPA pode ser um instrumento de rastreio sensível às alterações do processamento auditivo, embora não avalie as demais habilidades auditivas.
\end{abstract}

Palavras-chave: Percepção sonora. Córtex Auditivo. Vias Auditivas. Testes Auditivos. Audição

ABSTRACT
Introduction: The first years of a child's life are critical periods for speech and
language development, and they are fundamental to sociability of the human being.
During this period, the hearing abilities are developed due to the great maturation and
auditory plasticity of the central nervous system. Thus, a good development of the
hearing abilities will be favorable in the learning process in preschool and school 
children. Objective: To investigate the hearing abilities of the first and second year of elementary school students of nine years through the Simplified Auditory Processing Test (SAPT) performance. Methodology: The casuistry consisted of 67 schoolchildren enrolled regularly in the initial years at the Municipal School Hermes Vezzaro in the Municipality of Cascavel-PR, divided into two groups. Group 1 (G1), comprised of 51 first-year students aged 6, from this group 30 female and 21 males. Group 2 (G2), composed of 16 students of the second year aged 7 years old, 10 female and 6 male. All of them were submitted to the following procedures: anamnesis, inspection of the external auditory meatus, Screening audiometry and the tests that compose the Simplified Auditory Processing Test (SAPT). Results: Schoolchildren from both groups presented satisfactory performance in all tests, mostly showing attention abilities, sound localization and sequential memory. However, G1 provided lower responses when compared to G2. Younger children had a higher occurrence of changes in the Sequential Memory test for Non-Verbal Sounds. Conclusion: The majority of schoolchildren presented the attention abilities, sound localization and sequential memory adequate for the age group. SAPT can be a screening instrument sensitive to auditory processing changes, although it does not evaluate the other auditory abilities

Keywords: Sound perception. Auditory Cortex. Auditory pathways. Hearing Tests. Hearing

\section{INTRODUÇÃO}

Nos primeiros anos de vida de uma criança, ocorre o desenvolvimento das habilidades auditivas e de linguagem, sendo considerado um período crítico. Durante esse período acontece a maturação do sistema nervoso auditivo central, onde há maior plasticidade, permitindo o estabelecimento de novas conexões neurais que são imprescindíveis para o desenvolvimento normal da audição e da linguagem (MOMENSOHN-SANTOS e RUSSO, 2007).

O funcionamento normal dos processos auditivos, tanto periférico quanto central, permite que funções como receber, transmitir, perceber, relembrar os sons e integrar as experiências sonoras, proporcione a comunicação, além da interação social (KAMINSKI, TOCHETTO e MOTA, 2006). Com isso, torna-se fundamental acompanhar o processo da audição de uma criança em desenvolvimento, principalmente em idade escolar (MUSIEK e RINTELMANN, 2004; KATZ e TILLERY, 1997).

A maturação das habilidades auditivas contribui para a qualidade do processo de aprendizagem, principalmente em pré-escolares e escolares, pois a maneira como percebemos e entendemos a mensagem que está sendo transmitida torna-se um fator crucial para o desenvolvimento tanto da linguagem oral quanto da leitura e escrita. Nesse sentido, para um bom desempenho no processo de alfabetização, faz-se necessário que as habilidades de detecção, discriminação, localização, reconhecimento e compreensão dos sons estejam íntegras, assim como a sensação, memória para os sons e atenção, sendo esses correspondentes aos processos que envolvem o Processamento Auditivo Central (PAC) (ASHA, 1996).

As habilidades auditivas envolvidas no PAC são: detecção, localização sonora e lateralização, discriminação auditiva, reconhecimento, aspectos auditivos temporais, resolução, mascaramento, integração, ordenação, desempenho auditivo diante de estímulos sonoros competitivos e estímulos sonoros degradados (BELLIS, 1997). 
Sendo assim, a falta de habilidade do indivíduo em discriminar, reconhecer, atender, recordar e/ou compreender informações ouvidas, mesmo em níveis de inteligência normal e audição periférica podem ocorrer devido aos Transtornos do Processamento Auditivo Central (TPAC) (KEITH e PENSAK, 1991).

Estudos destacaram que as principais etiologias dos transtornos de processamento auditivo poderiam ser devido a atraso de maturação do sistema nervoso central, alterações das condições neurológicas e coexistência com outras disfunções do desenvolvimento (BAMIOU, MUSIEK E LUXON 2001).

Keith (1988) citou algumas características de indivíduos com TPAC, tais como: dificuldades em seguir ordens verbais, distração, fadiga em tarefas complexas ou prolongadas, necessidade de repetição frequente de estímulos verbais, aprendizado lento das relações grafo fonêmicas, alterações mnésicas, dificuldades em compreender piadas e linguagem figurada, além de sensibilidade exagerada frente a sons intensos.

Katz e Wilde (1989) relatam que os transtornos de aprendizagem, principalmente os de leitura, compreensão leitora, dificuldades com fonemas, habilidades com língua estrangeira e os comprometimentos de ortografia estão associados aos TPAC.

A atuação da Fonoaudiologia na escola tem como objetivo detectar e prevenir, por meio das triagens, os distúrbios da comunicação e também oferecer orientações aos professores, pais e demais integrantes da equipe escolar (CHARONE e NEGRÃO, 2001). Dividir conhecimentos relacionados à prevenção e desenvolvimento da audição, bem como consequências dos problemas auditivos proporciona uma série de benefícios ao ambiente escolar (MARANHÃO, PINTO e PEDRUZZI, 2009).

No entanto, comumente os procedimentos de triagem auditiva no âmbito escolar se limitam a avaliar a funcionalidade periférica, em virtude da facilidade no emprego e interpretação destes testes, tornando os procedimentos que avaliam as habilidades auditivas um desafio, pois muitos não têm acesso a uma avaliação mais específica da audição.

Neste contexto, existe uma preocupação na área da Audiologia quanto às formas de triagens, seja por meio de uma bateria de exames, ou através de questionários que possam buscar informações do PAC de maneira precoce, sendo esses, procedimentos de baixo custo e com maiores possibilidades de acesso.

Neves e Schochat (2005) afirmam que alterações em habilidades de localização sonora, memória sequencial para sons verbais e não verbais têm estreita relação com alterações de linguagem e aprendizagem, e essas habilidades podem ser avaliadas por meio do protocolo de Avaliação Simplificada do Processamento Auditivo (ASPA).

A ASPA é um dos procedimentos padronizados que vem sendo estudado em crianças e professores nos anos iniciais como alternativa para observar 0 comportamento das habilidades auditivas de atenção, localização, memória, processamento temporal e interação binaural. Esse procedimento sugere precocemente quaisquer possíveis dificuldades do PAC, já que essas habilidades são pré-requisitos para o desenvolvimento das demais funções auditivas.

A Avaliação Simplificada do Processamento Auditivo (ASPA) é de fácil aplicabilidade, a campo livre, ou seja, não necessita de uma cabina acústica nem de aparelhos sofisticados, sendo indicada para ser utilizada como triagem do PAC, principalmente nas escolas. 
Contudo, considerando que uma adequada maturação das habilidades auditivas contribui positivamente no processo de ensino-aprendizagem, e que os TPAC podem acarretar em atrasos no desenvolvimento da linguagem e cognição da criança, tornam-se necessários procedimentos que não se limitam em apenas avaliar a audição periférica, pois ouvir e entender as informações ouvidas demanda de uma complexidade maior, para além da parte periférica.

Sendo assim, a avaliação do PAC em crianças na fase escolar, em que está acontecendo o aprendizado constante, colabora para o diagnóstico precoce dos TPAC possibilitando, dessa forma, uma orientação pedagógica e terapêutica mais adequada (AITA et al, 2003).

Diante do exposto, o objetivo do presente estudo foi investigar as habilidades auditivas dos escolares do primeiro e segundo ano do Ensino Fundamental de nove anos por meio do desempenho nos testes da ASPA.

\section{METODOLOGIA}

Este estudo caracterizou-se como pesquisa de campo transversal, com abordagem quantitativa e descritiva.

Primeiramente foi realizada uma reunião com a diretora da Escola Municipal Hermes Vezzaro, localizada no Município de Cascavel - PR, onde foram explicados os objetivos do estudo, como também o desenvolvimento metodológico da pesquisa. Posteriormente, convidou-se a instituição de ensino a participar e, após a autorização e parecer do comitê de ética, foi encaminhado aos pais e/ou responsáveis o Termo de Consentimento Livre e Esclarecido, juntamente com o material explicativo e um questionário. Em seguida, foram convidados os alunos autorizados pelos pais ou responsáveis a participar e, os que concordaram, assinaram 0 Termo de Assentimento.

A casuística foi composta por 67 (sessenta e sete) escolares matriculados regularmente nos Anos Iniciais do Ensino Fundamental, sendo divididos em dois grupos: Grupo 1 (G1) e Grupo 2 (G2). O G1 foi formado por 51 (cinquenta e um) escolares do primeiro ano, 30 (trinta) do gênero feminino e 21 (vinte e um) do gênero masculino, com 6 (seis) anos de idade. O G2 foi composto por 16 (dezesseis) escolares do segundo ano, 10 (dez) do gênero feminino e 6 (seis) do gênero masculino, com 7 (sete) anos de idade.

Dentre os critérios de inclusão foram considerados alunos do $1^{\circ}$ e $2^{\circ}$ ano regularmente matriculados na escola já citada, com idades de 6 a 7 anos respectivamente, devidamente autorizados pelos responsáveis legais a participar da pesquisa mediante assinatura do Termo de Consentimento Livre e Esclarecido. Os alunos que voluntariamente quiseram participar da coleta de dados também assinaram o Termo de Assentimento.

Além disso, deveriam possuir condições ideais de Meato Acústico Externo e limiares auditivos dentro dos padrões de normalidade bilateralmente. Foram excluídos os escolares que não possuíam idades de 6 e 7 anos, ou que apresentaram diagnóstico de alteração neurológica, psicológica ou algo que pudesse interferir no teste, além de impedimentos no Meato Acústico Externo e variação dos limiares de audibilidade.

Todos os participantes foram submetidos aos seguintes procedimentos: questionário, inspeção visual do Meato Acústico Externo, Triagem Audiométrica e aplicação da ASPA. 
O questionário utilizado foi o proposto por Pereira \& Schochat, constituído de 25 questões objetivas referentes aos aspectos auditivos de linguagem e aprendizagem de seus (as) filhos (as), tendo como opções de respostas as alternativas "sim", "não" e "observações" (Anexo 3). O questionário foi enviado aos responsáveis juntamente com os termos e o material explicativo do presente estudo.

A Inspeção Visual do Meato Acústico Externo foi realizada com o objetivo de verificar a existência de impedimentos para realização da avaliação, sendo que os indivíduos que apresentaram alteração foram encaminhados ao médico otorrinolaringologista para avaliação e conduta. O otoscópio utilizado foi o modelo Mini Led 1000, da marca Mikatos.

Para realização da triagem audiométrica, utilizou-se o audiômetro modelo AD 229b, da marca Interacoustic, adaptado aos fones TDH-39, calibrado de acordo com a norma ISSO 8253-1 como referência para níveis de ruído máximo permitido, sob o número do certificado de calibração número 9982, de 06/02/2018 e corretamente posicionado na orelha do aluno. Para a verificação dos limiares de audibilidade foi utilizada a técnica descendente (estímulo aplicado de maneira audível para inaudível). O limiar de audibilidade foi obtido pela confirmação de $50 \%$ das respostas sobre quatro estímulos específicos (tom puro) apresentado em uma mesma frequência. $O$ critério adotado para classificação das perdas auditivas quanto ao grau foi baseado na média tritonal das frequências de $500,1.000$ e $2.000 \mathrm{~Hz}$, segundo Lloyd e Kaplan (1978), ou seja, o aluno que apresentou média tritonal até 25 dBNA teve limiares auditivos dentro dos padrões de normalidade, sendo os resultados superiores considerados alterados. O estímulo utilizado foi o tom puro de forma contínua, testadas as frequências de $500,1.000,2.000,3.000$ e $4.000 \mathrm{~Hz}$, respectivamente.

Posteriormente, foi realizada a Avaliação Simplificada do Processamento Auditivo (ASPA) composta por três testes: Teste de Localização Sonora, Teste de Memória Sequencial para Sons Verbais e Não Verbais.

O Teste de Localização Sonora (LS) em 5 direções (direita, esquerda, em cima, à frente e atrás), descrito por Pereira (1993), teve como objetivo avaliar a capacidade do escolar em localizar os sons. Tratou-se de uma tarefa diótica, de competência de localização sonora e integração binaural, sendo apresentado o som do guizo (instrumento sonoro de frequência aguda e de alta intensidade) numa distância de 20 cm para cada percussão.

Durante a apresentação dos estímulos, a criança permaneceu sentada de olhos fechados e, no final de cada som ouvido, ainda com os olhos fechados, teve de apontar ou nomear o local de origem do som. Os estímulos foram apresentados uma única vez em cada direção. Como critério de normalidade, a criança deveria acertar quatro das cinco direções dos sons.

O teste de Memória Sequencial para Sons Não Verbais (MSNV) foi descrito por Toniolo et al (1994) e teve como finalidade verificar a capacidade da criança memorizar sequencialmente e discriminar sons não verbais. Tratou-se de uma tarefa diótica de competência de processamento temporal, na qual foi apresentado o som de instrumentos sonoros (guizo, agogô, sino e coco) em três diferentes ordenações. Nos escolares de 6 anos utilizou-se três instrumentos, o guizo, sino e coco, nos alunos de 7 anos aplicou-se quatro estímulos, o guizo, coco, agogô e sino. Como critério de análise, os alunos de 6 anos tiveram que acertar pelo menos duas sequências ouvidas, e os de7 anos, três sequências.

O teste de Memória Sequencial Verbal (MSV) foi descrito por Toniolo et al (1994) e teve como intenção averiguar a capacidade do aluno em memorizar sequencialmente e discriminar sons verbais. Tratou-se de uma tarefa diótica com 
competência de processamento temporal, sendo utilizadas as sílabas $|\mathrm{Pa}|,|\mathrm{Ta}|,|\mathrm{Ca}|$, $|\mathrm{Fa}|$ nos escolares de 7 anos, e $|\mathrm{Pa}|,|\mathrm{Ta}|,|\mathrm{Ca}|$ nos escolares de 6 anos. Como critério de referência foi considerado a capacidade de ordenar duas sequências ouvidas (escolares de 6 anos) e três sequências (escolares de 7 anos).

Todos os procedimentos foram realizados individualmente, em uma sala silenciosa, dentro do ambiente escolar, com tempo de aproximadamente 20 minutos por aluno.

O presente estudo foi aprovado pelo Comitê de Ética do Centro Universitário Fundação Assis Gurgacz, conforme parecer 2.761.630 e CAAE: 90384518.3.0000.5219.

\section{RESULTADOS E DISCUSSÃO}

Por meio da análise dos resultados, pôde-se observar que as queixas mais citadas pelos pais ou responsáveis do G1 foram: desatenção, complicações no aprendizado da leitura e escrita, histórico de episódios repetitivos de otite média nos primeiros anos de vida, dificuldade de compreensão em ambientes ruidosos e problemas com a fala. No G2 as queixas apontadas foram: episódios repetitivos de otite média nos primeiros anos de vida, desatenção, complicações no aprendizado da leitura e escrita, dificuldade de concentração em ambientes ruidosos, além de problemas relacionados com a fala, conforme demonstrou a figura 1. Esses achados foram semelhantes ao estudo de Piletero, Manfred e Schneck (2010), que mencionam haver maior número de queixas quanto à dificuldade de ouvir em ambientes ruidosos, desatenção, demora na compreensão da conversação, problemas de memória e história de otites de repetição.

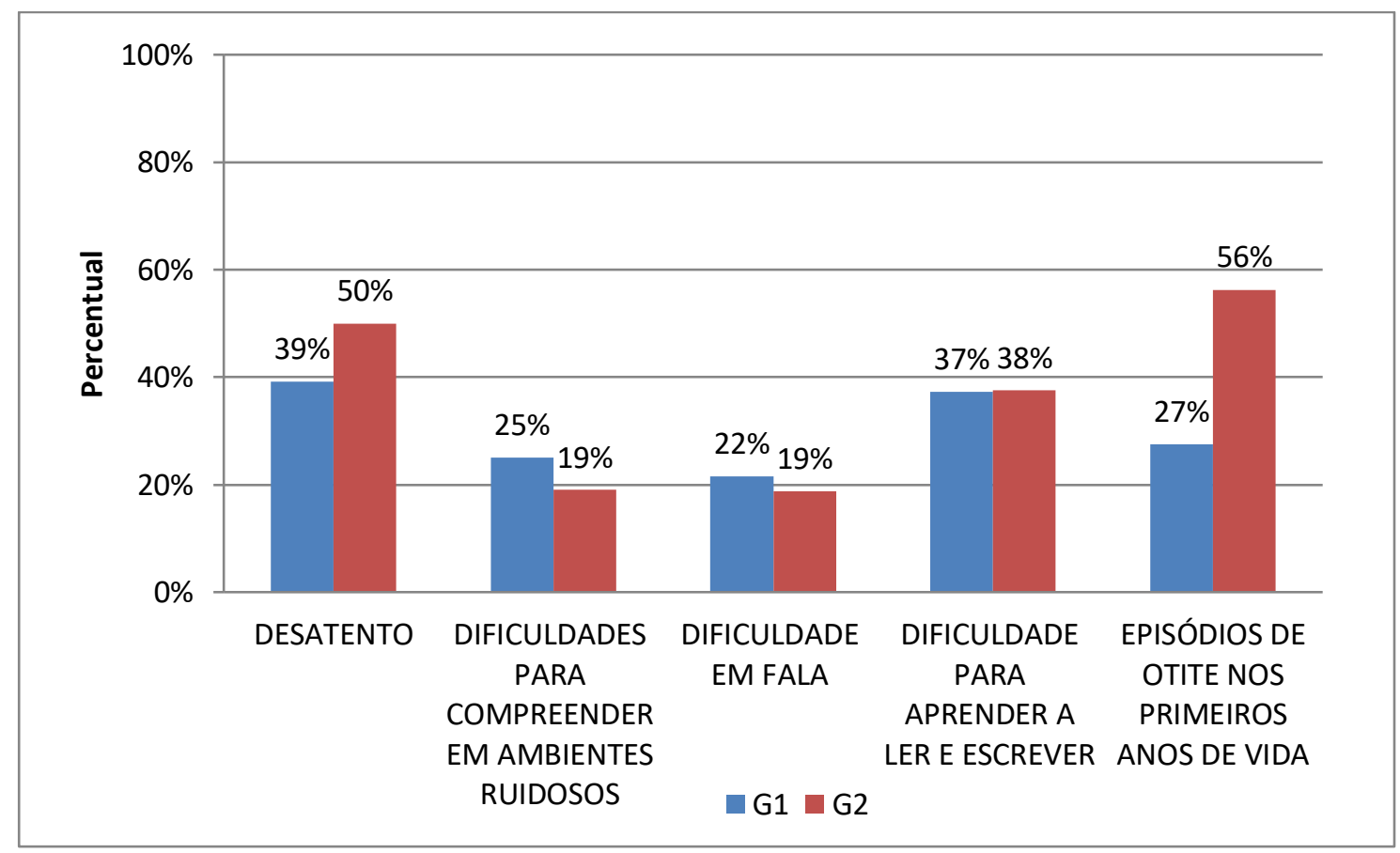

Figura 1. Distribuição percentual das queixas relatadas pelos responsáveis dos escolares de acordo com o G1 e G2.

Campos (2002) afirma que a otite é uma doença altamente prevalente na infância, com pico de incidência entre seis a vinte e quatro meses de idade, 
apresentando um segundo pico de incidência entre quatro e sete anos. Isto ocorre devido ao desequilíbrio dos mecanismos de proteção, aeração e drenagem na orelha média, ocasionada pela inclinação da tuba auditiva de $10^{\circ}$ em relação ao eixo horizontal, além do comprimento da tuba ser incompleto nesta faixa etária. Esses fatores fazem com que as crianças apresentam refluxo das secreções da nasofaringe para a orelha, favorecendo problemas inflamatórios e infecciosos de orelha média. Sendo assim, estudos apontam haver relação entre a otite média e o desempenho das habilidades do processamento auditivo central (PEREIRA, 1993; TEDESCO, 1997; BALBANI e MANTOVANI, 2003; KLAUSEN et al, 2003; BOSCARIOL, ANDRÉ e FENIMAN, 2009).

Em contrapartida, Santos et al (2001) asseguram não haver diferença estatisticamente significante entre o desempenho das crianças com e sem histórico de otite média nos testes comportamentais de localização sonora, teste de memória para sons verbais e não verbais em sequência, teste de fala com ruído branco, teste dicótico de dígitos, teste dicótico não verbal e teste PSI em português.

Neste estudo foram observadas queixas referentes a dificuldades no aprendizado da leitura e da escrita, entretanto, de acordo com a Base Nacional Comum Curricular (2017), o processo de alfabetização das crianças deve ocorrer até o final do segundo ano do Ensino Fundamental. Nesse sentido, considerando que os escolares estudados encontram-se nos anos Iniciais do Ensino Fundamental de nove anos, não se pode caracterizar especificamente essa queixa como uma dificuldade no processo de aprendizagem da leitura e escrita, porém nos alerta para a necessidade de acompanhá-los quanto às noções consideradas como pré-requisitos no desenvolvimento da leitura e escrita. Todavia, Toniolo (1994) reforçou que crianças com queixa de dificuldades no aprendizado escolar podem apresentar TPAC decorrentes de alterações nas habilidades auditivas, tais como: localização, atenção, figura-fundo, memória, discriminação, análise e síntese.

Quanto à queixa de dificuldade na fala, estudos alegaram haver relação dos problemas de fala com os TPAC (PEREIRA, 1996; KAMINSKI, TOCHETTO e MOTA, 2006; MUNIZ et al, 2007; RABELO et al, 2015; SOUZA, PASSAGLIO e LEMOS, 2016). Ziliotto et al (2002) estudaram dois casos clínicos de crianças em idade pré-escolar e com distúrbio na produção articulatória que foram submetidas à avaliação do PAC, cujos resultados revelaram-se alterados. As autoras concluíram então que uma criança com alteração na fala deve passar pelo diagnóstico das habilidades auditivas.

Pereira (1996) averiguou que as substituições na fala envolvendo o fonema /r/ e ///, inversões de letras, dificuldades na compreensão leitora e dificuldades de atenção em ambientes ruidosos são exemplos das principais manifestações comportamentais do TPAC.

Hall e Mueller (1997) afirmam serem queixas comuns de indivíduos com TPAC dificuldade nas áreas de leitura e ortografia; problemas em manter a atenção; dificuldade em permanecer na mesma atividade, como completar uma tarefa ou trabalho; histórico de episódios de otite; dificuldade em associar letras do alfabeto aos respectivos sons; dificuldade em associar sons às fontes que os produziram; vocabulário pobre; hiperatividade ou hipoatividade; desempenho inferior em testes que exigem compreensão oral-verbal; lentidão para responder a informações auditivas; distração e comportamentos inadequados.

Em relação à ASPA, pôde-se observar que os escolares de ambos os grupos apresentaram desempenho satisfatório em todos os testes. No entanto, o G1 apresentou respostas inferiores quando comparados ao G2, pois, dos 51 escolares 
pertencentes ao G1, 2 (4\%) mostraram dificuldade nos testes de LS e MSV, enquanto $4(8 \%)$ apresentaram alteração no teste MSNV. Já os 16 alunos do G2 obtiveram resultados normais em todos os testes, de acordo com a figura 2.

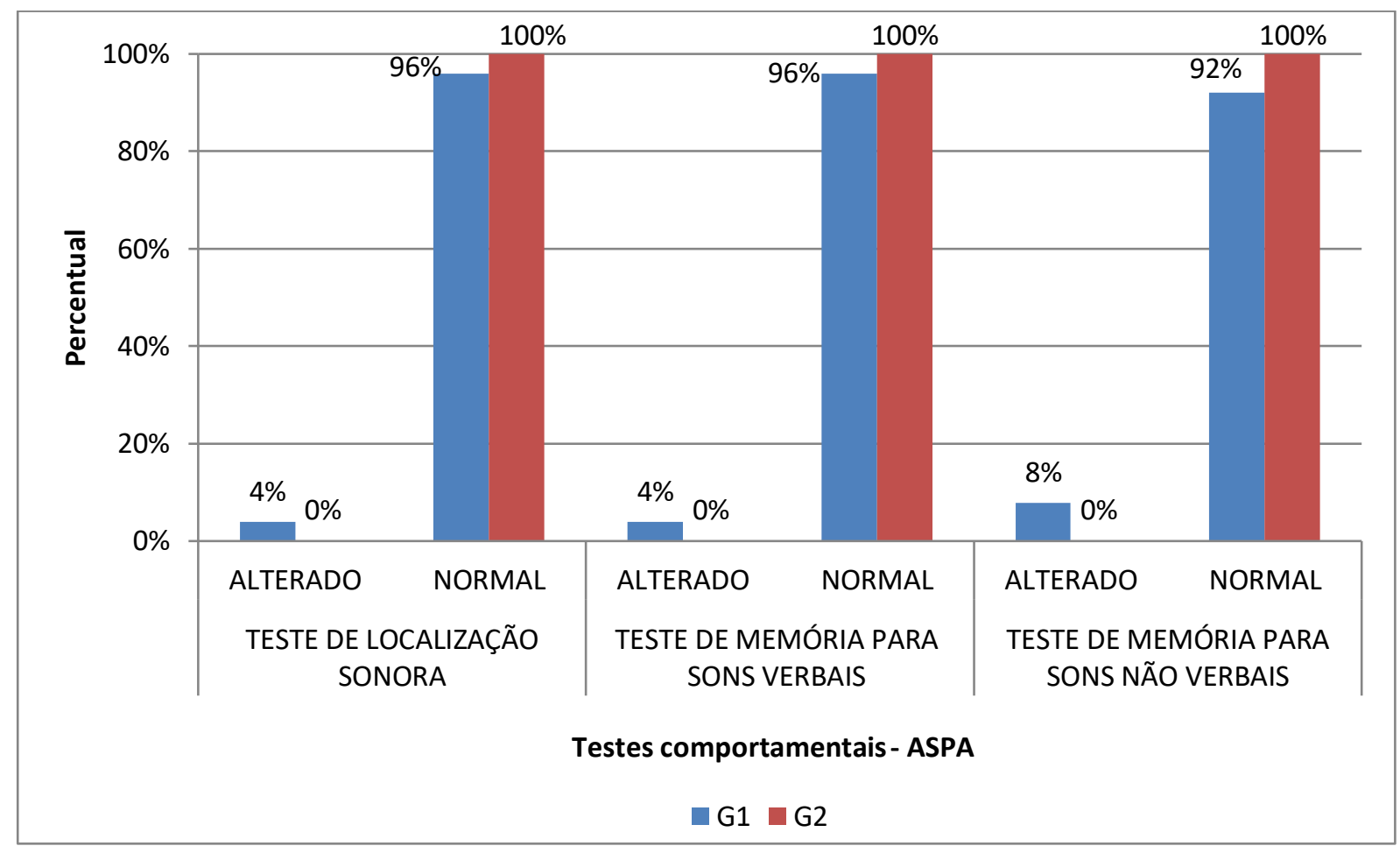

Figura 2. Distribuição percentual dos resultados nos testes da ASPA de ambos os grupos.

Os achados do teste de LS foram semelhantes aos encontrados no estudo de Santos et al (2008), pois identificaram que $95,7 \%$ dos escolares de oito a treze anos de idade apresentaram condições de normalidade para o teste de LS. Etges et al (2011) também verificaram que dos 130 alunos de 7 a 10 anos de idade, 93,11\% passaram no teste de LS em cinco direções.

Os resultados do teste MSV corroboram com os estudos de Santos et al (2008) e Vale (2009), pois os autores apontaram resultados satisfatórios para a faixa etária estudada.

Pôde-se perceber pior desempenho no teste MSNV, sendo que esse resultado se assemelha ao estudo de Meneguello et al (2001) que, após analisarem 100 protocolos de avaliações do Processamento Auditivo Central (PAC) de indivíduos de ambos os gêneros e com idades entre 7 a 18 anos, concluíram que a memória sequencial foi a habilidade auditiva mais frequentemente alterada na população. Etges et al (2011) mencionam que $40,16 \%$ dos alunos avaliados obtiveram resultados positivos neste teste.

Em relação à associação dos testes da ASPA com dificuldades na linguagem oral, bem como na aprendizagem escolar, Cruz e Pereira (1996) verificaram que as crianças avaliadas com alteração na linguagem também apresentaram desempenho inferior nos testes da ASPA. Vale (2009) relatou haver correlação entre as dificuldades de leitura com as alterações nos testes da ASPA.

Observou-se que todas as alterações encontradas nos testes aconteceram no G1, sendo que os escolares pertencentes ao G2 apresentaram 100\% de normalidade. Estudos demonstraram que crianças mais novas quando comparadas com idades 
superiores apresentaram um percentual de falha maior nos testes da ASPA, ressaltando a importância do acompanhamento das habilidades auditivas durante 0 período pré-escolar e escolar (CORONA et al, 2005; COLELLA-SANTOS et al, 2009).

$O$ fato de a maioria dos escolares ter apresentado resultados satisfatórios nos testes da ASPA, sugere que o desenvolvimento dessas habilidades auditivas encontra-se dentro dos padrões ideais para a faixa etária, sendo relevante para o desenvolvimento acadêmico. Por sua vez, os escolares que falharam nos testes podem estar no período de maturidade dessas funções. Nessa perspectiva, o contato da criança com o mundo sonoro, bem como as experiências vividas por ela no meio familiar e escolar, proporcionam o aumento do processo de mielinização das fibras nervosas, ocasionando um melhor desenvolvimento das habilidades de interpretação e análise dos padrões sonoros (PEREIRA, 1993; COLELLA-SANTOS et al, 2009). Além disso, as habilidades auditivas vão se desenvolvendo com o crescimento, tornando-se próximas do adulto entre a faixa etária de 10 a 11 anos (MUSIEK e RINTELMANN, 2001). Entretanto, não se deve descartar a possibilidade de acompanhamento desses escolares, visando à detecção precoce de possíveis TPAC, com intuito de minimizar suas manifestações, seja na linguagem oral, aprendizagem escolar, questões sociais, dentre outras.

Nesse sentido, a triagem das habilidades auditivas tem grande relevância no período pré-escolar e escolar, uma vez que, o processamento da informação auditiva está intimamente conexo com o desenvolvimento da fala, dos aspectos cognitivos e de aprendizagem escolar.

Contudo, a ASPA, por ser um procedimento simples que pode ser utilizada em diferentes faixas etárias, além de ser realizada em campo livre, no próprio ambiente escolar e sem necessidade de cabina acústica, torna-se um importante instrumento de rastreio para sugerir possíveis alterações das habilidades auditivas, pois, além de proporcionar direcionamento e acompanhamento precoces aos indivíduos que falharam na triagem, oferece a possibilidade de orientação tanto aos familiares quanto à equipe pedagógica. Porém, o procedimento ASPA não é o suficiente para o diagnóstico dos TPAC, sendo importante e necessário o encaminhamento para avaliação completa do PAC.

\section{CONSIDERAÇÕES FINAIS}

Com base na análise dos resultados, pôde-se concluir que a maioria dos escolares possuem habilidades de atenção, localização sonora e memória sequencial adequadas para a faixa etária. As crianças mais jovens apresentaram maior ocorrência de alterações no teste de Memória Sequencial para Sons Não Verbais.

Tendo em vista a estreita relação entre as habilidades auditivas e a aquisição da aprendizagem, tornam-se importantes procedimentos de triagens do processamento auditivo em pré-escolares e escolares. Desta forma, a ASPA pode ser um instrumento de rastreio sensível às alterações do processamento auditivo, embora não avalie todas as habilidades auditivas.

\section{REFERÊNCIAS}

AITA, A.D.C.; MESQUITA, C.D.S.; CAMPOS, C.M.; FUKUDA, M.T.H.; AITA, F.S. Correlação entre as desordens de processamento auditivo central e queixas de dificuldades escolares. Jornal Brasileiro de Fonoaudiologia. v. 4, n. 5, p. 101-107, 2003. 
AMERICAN SPEECH-LANGUAGE-HEARING ASSOCIATION. (ASHA). Central Auditory Processing: current status of research and implications for clinical practice. American Journal of Audiology. v. 5, n. 2, p. 41-54, 1996.

BALBANI, A.P.S.; MONTOVANI, J.C. Impacto das otites médias na aquisição da linguagem em crianças. Jornal de Pediatria. v. 79, n. 5, 2003.

BAMIOU, D.E; MUSIEK, F.E; LUXON, L.M. Aetiology and clinical presentations of auditory processing disorders - a review. Arch Dis Child. v. 85, n.5, p. 361-365, 2001.

BELLIS, T.J. Assessment and management of central auditory processing disorders in the education setting: from science to practice.2th ed. San Diego, CA: Singular Publishing Group, 1997.

BOSCARIOL, M.; ANDRÉ, K.D.; FENIMAN, M.R. Crianças com fissura isolada de palato: desempenho nos testes de processamento auditivo. Brazilian Journal of Otorhinolaryngology. v. 75, n. 2, p. 213-220, 2009.

BRASIL. Base Nacional Comum Curricular (BNCC). Educação é a Base. Brasília, MEC/CONSED/UNDIME, 2017. Disponível <http://basenacionalcomum.mec.gov.br/> Acesso em: 19 nov. 2018.

em:

CAMPOS, C.A.H. Tratado de otorrinolaringologia. São Paulo: Roca; 2002.

CHARONE, A.; NEGRÃO, A. A fonoaudiologia na escola. Revista Lato \& Sensu, Belém, v. 2, n. 4, p. 7-9, 2001.

COLELLA-SANTOS, M.F.; BRAGATTO, G.R.; MARTINS, P.M.F.; DIAS, A.B. Triagem auditiva em escolares de 5 a 10 anos. Revista CEFAC. v. 11, n. 4, p. 644-653, 2009. 